\title{
Intra-cavity Photodynamic Therapy for malignant tumors of the paranasal sinuses: An in vivo light dosimetry study
}

\author{
T.E.M. van Doeveren ${ }^{\mathrm{a}, \mathrm{b}}$, R.L.P. van Veen ${ }^{\mathrm{a}}$, F. van den Boom ${ }^{\mathrm{c}}$, I.B. Tan ${ }^{\mathrm{a}, \mathrm{d}, \mathrm{e}, \mathrm{f}}$, W.H. Schreuder ${ }^{\mathrm{a}, \mathrm{g}}$, \\ M.B. Karakullukçu ${ }^{\mathrm{a}, \mathrm{g}, *}$ \\ a Department of Head and Neck Oncology and Surgery, Verwelius lab, The Netherlands Cancer Institute, Antoni van Leeuwenhoek Hospital, Amsterdam, the Netherlands \\ ${ }^{\mathrm{b}}$ Department of Otolaryngology and Head and Neck Surgery, Erasmus University Medical Center, Rotterdam, the Netherlands \\ ${ }^{\mathrm{c}}$ Department of Radiation Oncology, The Netherlands Cancer Institute, Antoni van Leeuwenhoek Hospital, Amsterdam, the Netherlands \\ ${ }^{\mathrm{d}}$ Department of Otorhinolaryngology and Head and Neck Surgery, Maastricht University Medical Center, Maastricht, the Netherlands \\ e GROW-School for Oncology and Developmental Biology, Maastricht University Medical Center, Maastricht, the Netherlands \\ ${ }^{\mathrm{f}}$ Department of Otolaryngology and Head and Neck surgery, Gadjah Mada University, Dr. Sardjito Hospital, Yogyakarta, Indonesia \\ ${ }^{g}$ Department of Oral and Maxillofacial Surgery, Academic Medical Centre/Academic Centre for Dentistry Amsterdam, University of Amsterdam, Amsterdam, the \\ Netherlands
}

\section{A R T I C L E I N F O}

\section{Keywords:}

Light dosimetry

Photodynamic therapy

Paranasal sinuses

In vivo light distribution

Fluence rate

Intra-cavity dosimetry

\begin{abstract}
A B S T R A C T
Background: Photodynamic therapy (PDT) is a promising treatment option for recurrent sinonasal malignancies. However, light administration in this area is challenging given the complex geometry, varying tissue optical properties and difficult accessibility. The goal of this study was to estimate the temporal and spatial variation in fluence and fluence rate during sinonasal mTHPC-mediated PDT. It was investigated whether the predetermined aim to illuminate with a fluence of $20 \mathrm{~J} \cdot \mathrm{cm}^{-2}$ and fluence rate of $100 \mathrm{~mW} \cdot \mathrm{cm}^{-2}$ was achieved.

Methods: In eleven patients the fluence and fluence rates were measured using in vivo light dosimetry at the target location during real-time sinonasal PDT. There was a variance in sinonasal target location and type of light diffuser used. In four patients two isotropic detectors were used within the same cavity.

Results: All measurements showed major fluence rate fluctuations within each single isotropic detector probe over time, as well as between probes within the same cavity. The largest fluence rate range measured was 328 $\mathrm{mW} \cdot \mathrm{cm}^{-2}$. Only one probe showed a mean fluence rate of $\sim 100 \mathrm{~mW} \cdot \mathrm{cm}^{-2}$. Taken all probes together, a fluence rate above $80 \mathrm{~mW} \cdot \mathrm{cm}^{-2}$ was measured in $31 \%$ of the total light exposure; in $22 \%$ it was less than $20 \mathrm{~mW} \cdot \mathrm{cm}^{-2}$. Thirty-three percent showed a fluence of at least $20 \mathrm{~J} \cdot \mathrm{cm}^{-2}$.

Conclusions: The current dosimetry approach for sinonasal intra-cavity PDT shows major temporal and spatial variations in fluence rate and a large variance in light exposure time. The results emphasize the need for improvement of in vivo light dosimetry and dosimetry planning.
\end{abstract}

\section{Introduction}

In order to induce an optimal treatment effect of Photodynamic Therapy (PDT) it is essential that the three basic components of PDT (i.e. photosensitizer, light and oxygen) come together in the appropriate amount at the target location. Since the development of PDT, research has been implemented to further improve the treatment, for example by photosensitizer advancement or by refinement of the light application by using light dosimetry [1-6]. Among many treatment variables, a too low, or conversely too high light fluence rate is associated with a reduced cell response and depletion of the photosensitizer or oxygen within the cells, which finally result in a reduced PDT effect and an inadequate tumor response [1-5,7]. If using the photosensitizer meta-tetraHydroxyPhenylChlorin (mTHPC or Foscan ${ }^{\circledR}$ ) in the treatment of head and neck tumors, a light dose of approximately 20 Joules $\cdot \mathrm{cm}^{-2}$ with a fluence rate of $100 \mathrm{mWatt} \cdot \mathrm{cm}^{-2}$ has been clinically proven to

Abbreviations: PDT, Photodynamic Therapy; 3D, three dimensional; mTHPC, meta-TetraHydroxyPhenylChlorin; SD, standard deviation; CT, computed tomography; MR, Magnetic Resonance.

* Corresponding author at: Netherlands Cancer Institute, Antoni van Leeuwenhoek hospital, department of Head and Neck Oncology and Surgery, Plesmanlaan 121, 1066 CX Amsterdam, the Netherlands.

E-mail address: b.karakullukcu@nki.nl (M.B. Karakullukçu). 
induce the desired clinical response [8-11].

For surface PDT the dosimetry (the delivered light dose (fluence) and fluence rate) is straightforward, based on the laser source output power $(\mathrm{mW})$ and target surface area $\left(\mathrm{cm}^{2}\right)$. The surrounding healthy tissue needs to be shielded by green cloth or black wax to prevent from unintended damage, because of back scattering of the light. Previously experiences with PDT in hollow organs such as the esophagus and bladder showed that the actual delivered fluence rate in a hollow cavity could be significantly higher than the primary fluence rate, due to reemitting and reflection of photons by the (healthy) tissue within the cavity. This compared to the straightforward surface PDT where the target area is completely isolated [12-17]. In these target areas, it is challenging, otherwise non-feasible to cover the surrounding healthy tissue that influences this back-scattering component. Light delivery applicators in conjunction with in vivo dosimetry have been developed for this purpose, to be able to illuminate these complex target areas such as the nasopharynx or lung $[18,19]$.

Several studies have investigated the use of PDT in the head and neck area over the past two decades, although, treatment of (recurrent) malignant tumors of the nasal cavity and paranasal sinuses (including the maxillary, ethmoid and sphenoid sinus and nasopharynx) is still fairly recent [8,9,20-22]. Malignant tumors of these cavities recur frequently after the 'golden standard' treatment, which is surgery and adjuvant (chemo)radiotherapy. The vicinity of essential structures (e.g. eyes, brain, cranial nerves etcetera) limits additional treatment options such as salvage surgery or re-radiation, where PDT is a potentially promising treatment option in case of recurrences [20,22]. The paranasal sinuses have a very complex geometry as for example compared to the oral cavity, trachea or bladder. In addition, endoscopic survey of these post-surgical cavities reveals a variety of tissue types at the cavity surface (e.g.. mucosa, bone, fibrosis, mucus, crusts etcetera), which result in variable tissue optical properties. It is therefore to be expected that these geometries create regions with over and under illuminated areas.

In this dosimetric pilot study, we aim to gain insight in the light delivery approach within the sinonasal cavity by measuring the fluence rate and fluence (i.e. light dose) in vivo at the target area within the nasal cavity, paranasal sinuses and nasopharynx during mTHPC-mediated PDT. The goal was to illuminate the target area with a fluence rate of $100 \mathrm{~mW} \cdot \mathrm{cm}^{-2}$ and to deliver a fluence of $20 \mathrm{~J} \cdot \mathrm{cm}^{-2}$, based on source output power and tissue surface area without taking back scattering into account.

\section{Materials and methods}

\subsection{Patients}

In situ dosimetry was performed in eleven patients (A-K) receiving mTHPC-mediated superficial PDT to treat (recurrent) sinonasal malignancies (see Table 1). All patients were treated between January 2015 and May 2017 in the Netherlands Cancer Institute/Antoni van Leeuwenhoek Hospital, Amsterdam, the Netherlands. The study was in accordance with the Helsinki declaration and approved by the medical ethics committee. Because of the $8-10 \mathrm{~mm}$ tissue penetration depth of the $652 \mathrm{~nm}$ light, the inclusion for PDT treatment was a tumor thickness of less than $5 \mathrm{~mm}$. Tumors with more than $5 \mathrm{~mm}$ thickness were first (partially) surgically resected prior to the PDT treatment. The time interval between the surgery and the PDT treatment was set to at least 6 weeks to allow the tissue to recover and to minimize any negative impact from the wound surface during PDT such as blood loss or crusts [22]. Fig. 1 shows the CT imaging of patient $C$ with a sphenoid sinus malignancy prior to $(A)$ and after $(B)$ the salvage surgery, previous to PDT. The photosensitizer mTHPC ${ }^{\circledR}$ (Biolitec Pharma, Bonn, Germany) was administered in a proximal arm vein with a dose of $0.15 \mathrm{mg} / \mathrm{kg}$ body weight according to the standard dose approved by the European Medicines Agency [23].
Table 1

An overview of the patients in whom light dosimetry measurements were performed in vivo during sinonasal PDT. The anatomical position of the target area, the number of isotropic detector probes used during the measurement within the target area and the type of light source diffuser (linear, spherical or microlens) are shown.

\begin{tabular}{|c|c|c|c|}
\hline Patient & $\begin{array}{l}\text { Target area (Tumor } \\
\text { location) }\end{array}$ & $\begin{array}{l}\text { Number of isotropic } \\
\text { detector probes }\end{array}$ & $\begin{array}{l}\text { Type of light } \\
\text { diffuser }\end{array}$ \\
\hline A & nasopharynx & 2 & linear \\
\hline B & $\begin{array}{l}\text { nasopharynx, sphenoid } \\
\text { sinus }\end{array}$ & 1 & linear \\
\hline C & $\begin{array}{l}\text { sphenoid sinus, anterior } \\
\text { skull base }\end{array}$ & 2 & spherical \\
\hline D & maxillary sinus & 1 & microlens \\
\hline E & maxillary sinus & 1 & spherical \\
\hline $\mathrm{F}$ & maxillary sinus & 1 & spherical \\
\hline G & $\begin{array}{l}\text { sphenoid sinus, anterior } \\
\text { skull base }\end{array}$ & 2 & spherical \\
\hline $\mathrm{H}$ & maxillary sinus & 2 & microlens \\
\hline I & nasopharynx & 1 & linear \\
\hline $\mathrm{J}$ & frontal sinus & 1 & microlens \\
\hline K & nasopharynx & 1 & linear \\
\hline
\end{tabular}

\subsection{Pre-planning of the PDT illumination}

In all patients, the target area for the light exposure was determined prior to the PDT, based on the findings during clinical examination combined with Computed Tomography (CT) or Magnetic Resonance (MR) imaging. A basic illumination plan was made previous to the PDT, based on the light source output power $\left(P_{\text {out }}\right.$ in $\left.\mathrm{mW}\right)$ together with the source location (source to target distance $r$ ) and size of the target surface area $(A)$. An attempt was made to determine the optimum distance between the center of the light source and the target area $\left(r\right.$ in $\left.\mathrm{cm}^{2}\right)$ using the CT and MR data. This in order to define the most favorable or 'optimal' light source position within the sinonasal cavity, to reach a fluence of $20 \mathrm{~J} \cdot \mathrm{cm}^{-2}$ and fluence rate of $100 \mathrm{~mW} \cdot \mathrm{cm}^{-2}$. In two patients it was decided before the PDT, to illuminate with a maximum fluence of 10 $\mathrm{J} \cdot \mathrm{cm}^{-2}$ to limit damage to critical surrounding structures, adjacent to the target area. Depending on the location and shape of the target area, it was determined which type of light source diffuser was the most applicable to access the target area. A linear diffuser was chosen to illuminate a luminal shaped target area. During illumination of the nasopharynx, the linear diffuser was inserted in the nasopharynx applicator [19]. To calculate the surface area $A$ in case of the linear diffuser, the equation $A=2 \cdot \pi r L$ was used. Whereas $L$ is the length of the target area. The output power of the linear diffuser was set at 100 $\mathrm{mW} \cdot \mathrm{cm}^{-2}$. A micro-lens diffuser, providing a feasible perpendicular illumination, was employed in the case of an easily accessible target area within the sinonasal cavity. The surface area was estimated according to the equation $A=\pi \cdot r^{2}$. If illumination with the micro-lens diffuser was not feasible, approximating a more concave shape and large target area, a spherical diffuser was used (all diffusers types; Medlight SA, Ecublens, Switzerland). To calculate the surface area for the spherical diffuser the equation $A=4 \cdot \pi \cdot r^{2}$ was used.

\subsection{PDT illumination and in situ dosimetry}

Four days after mTHPC administration, the PDT took place under general anesthesia at the operating theatre. A $2 \mathrm{~W}$ diode laser producing a continuous wave of light at $652 \mathrm{~nm}$ was used (Ceralas PDT, Biolitec, Bonn, Germany), which was calibrated in air in an incorporated integrating sphere. The output of the diode laser was set, dependent on the predetermined light diffuser used, with a range of 0.1 to $0.9 \mathrm{~W}$. The source was maneuvered manually under endoscopic surveillance by the surgeon to the preferable location as the prior estimated light source to target area surface distance $r$. Details on the target location and type of light diffuser used are given in Table 1 . If feasible, the healthy tissue 


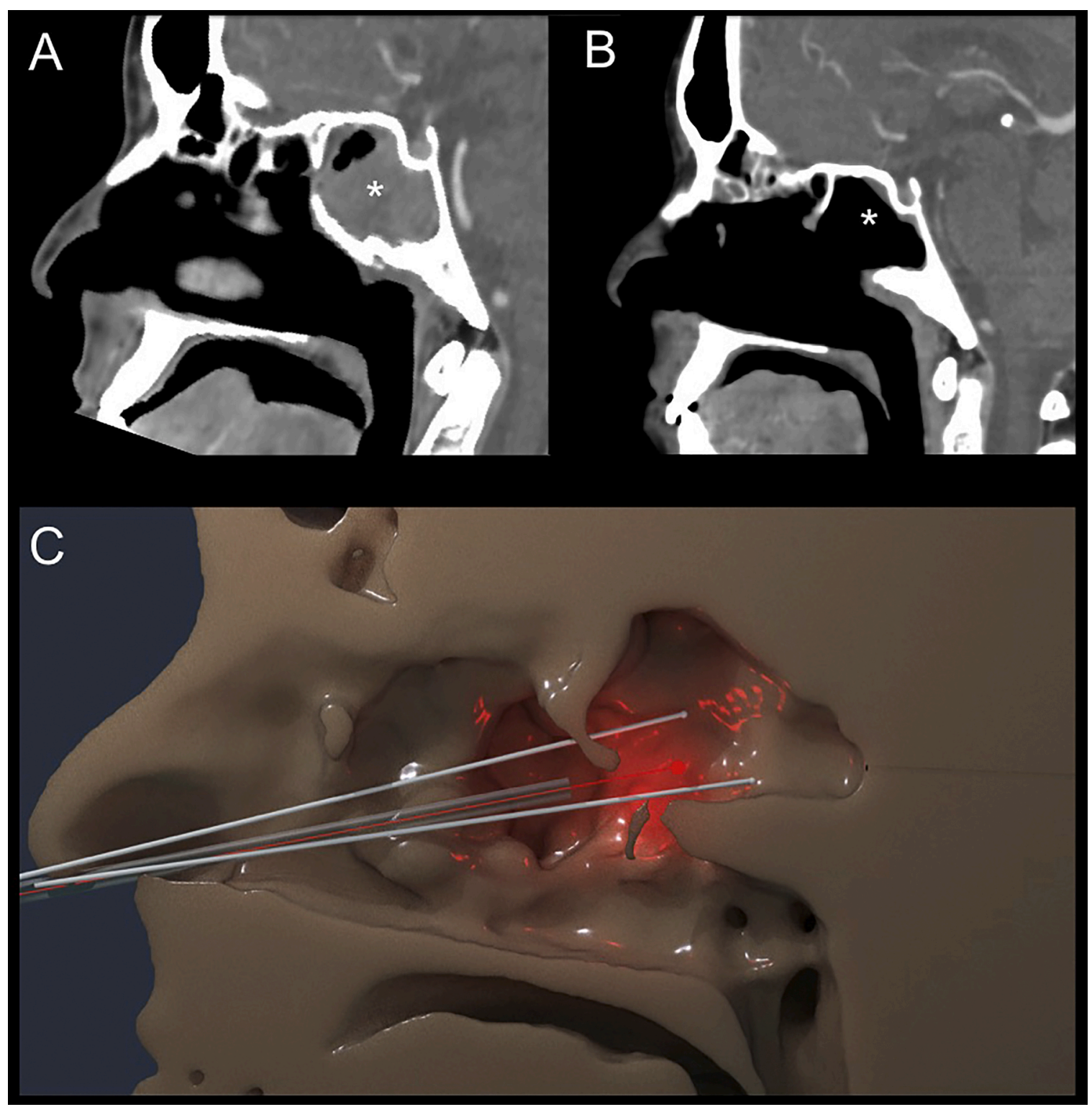

Fig. 1. (A) CT-scan in the sagittal plane of patient C with a tumor located in the sphenoid sinus (asterisk *) prior to any treatment. (B) CT-scan of the same patient after salvage surgery of the tumor, prior to PDT. The asterisk (*) shows the sphenoid sinus and the target area of the PDT. (C) 3D render reconstruction from the CT imaging of patient $\mathrm{C}$ prior to the PDT treatment. The white tubes represent the two isotropic detector probes. The red bulb indicates the spherical light diffuser with the light reflection on the sphenoid sinus surface.

surrounding the target area was covered with wet green drapes or black wax to prevent accidental light exposure, although in most locations covering was not possible, with the exception of the built-in covering of the nasopharynx applicator.

Real-time, during PDT illumination, the fluence and fluence rate on the surface of the target area were determined in vivo by using a dosimetry device (NI PXI-1045 18-slot Chassis, National Instruments) [19]. Isotropic light detector probes with a $0.865 \mathrm{~mm}$ distal tip diameter and a $400 \mu \mathrm{m}$ core diameter (model IP85, Medlight S.A., Ecublens, Switzerland) were connected to the device. To protect the fragile spherical tip, the isotropic probes were inserted into a proguide brachy catheter (Proguide Round Needle Set, 6 F, Elekta, Stockholm, Sweden) The isotropic detector probes measure the total fluence rate, i.e. the direct irradiance emitted by the light source together with the fluence rate caused by tissue back scattering within the cavity. Prior to the measurements the isotropic detector probes were calibrated in air, making use of an integrating sphere illuminated by baffle shielded light emitting diodes (LED's), generating a homogeneous stable reference fluence rate field. The dosimetry device (LabVIEWTM) gives an overview of the fluence rate and fluence plotted over light exposure time per single isotropic detector probe. Depending on the perioperative space, one or two isotropic detector probes were placed manually by the surgeon in the target area. The probes were hand-held by the surgeon at the same location during the illumination to yield a value for total fluence delivered at the spot investigated. In Fig. 1C a 3 dimensional render reconstruction of the dosimetry measurement with two isotropic detector probes during PDT illumination is shown. This reconstruction is based on the CT imaging of patient C. An example of the PDT and dosimetry measurement set up in the operation room is shown in Fig. 2. As shown in Fig. 2A and B the sinonasal cavity has an irregular shape and can be small to work in with the surgical tools.

\subsection{Data analysis}

The dosimetry device measures and stores the fluence rate, fluence ( $i$. $e$ light dose) and light exposure time per single isotropic detector probe 


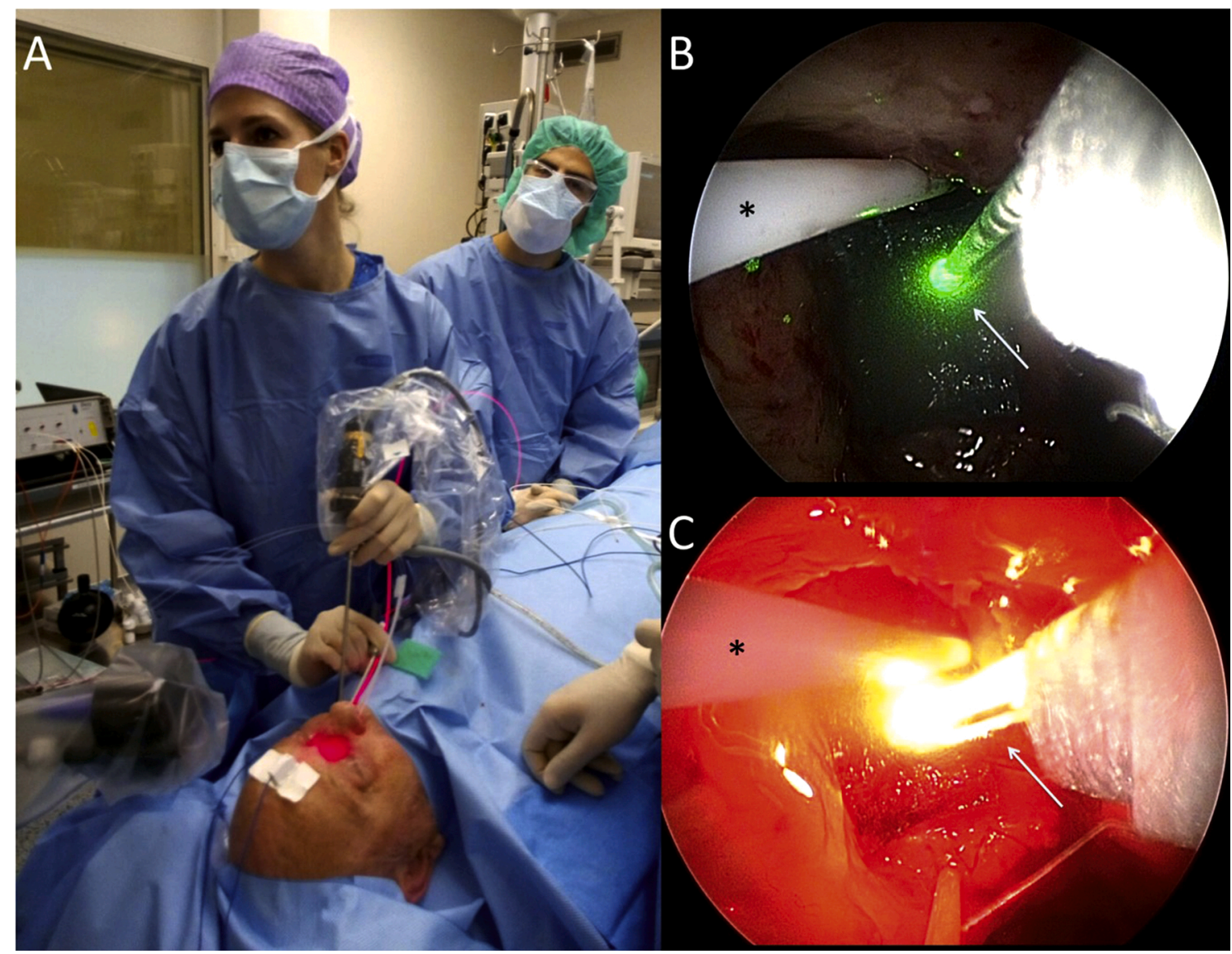

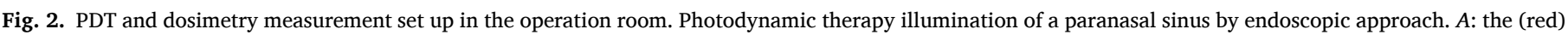

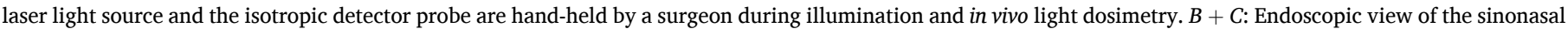

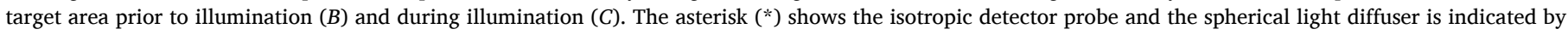
the arrow.

per second. The fluence rate and fluence measurements were analyzed for each single probe. If the illumination was interrupted (e.g. for repositioning of the light source or in case of source or shielding shifts during the manually guided illumination; to secure the healthy tissue shielding) this time and associated data were excluded from the calculations. The fluence rate's mean, standard deviation (SD) and range i.e. maximal and minimal values were calculated. Every measured fluence rate over time per single probe was divided into intervals of 40 $\mathrm{mW} \cdot \mathrm{cm}^{-2}$. The percentage of the total light exposure time measured within the interval was calculated. In order to determine the spatial variation, a description of the light exposure per isotropic detector probe was performed in the patients with two probes positioned within the same cavity during illumination. A descriptive analysis was chosen in the absence of enough data for statistical substantiation.

\section{Results}

Multiple types of target locations were treated including the nasopharynx, maxillary sinus, sphenoid sinus, frontal sinus, as well as the anterior skull base. In four patients the fluence rate and fluence was measured by using two isotropic detector probes at two different locations within the cavity. In seven patients we used a single isotropic detector probe. There was a large variability in type of light diffuser used, the laser output and light exposition time. An overview of the target area location, number of isotropic detector probes and type of light diffuser is shown in Table 1. The goal was to illuminate until a fluence of $\sim 20$ $\mathrm{J} \cdot \mathrm{cm}^{-2}$ was achieved, therefore the illumination duration varied for each individual PDT session. In five probes in four different patients the total measured fluence was around $10 \mathrm{~J} \cdot \mathrm{cm}^{-2}$ because of different reasons, including a too long light exposure time to reach the $20 \mathrm{~J} \cdot \mathrm{cm}^{-2}$ or a second probe which reached a total dose of $20 \mathrm{~J} \cdot \mathrm{cm}^{-2}$ earlier. Besides, in for example patient $\mathrm{C}$, a total light dose of $10 \mathrm{~J} \cdot \mathrm{cm}^{-2}$ was deliberately chosen to limit the risk of cerebrospinal fluid leakage when illuminating the anterior skull base. In two patients (two isotropic detector probes) a total light dose far above the $20 \mathrm{~J} \cdot \mathrm{cm}^{-2}$ was measured. This total light dose was high because multiple target areas were all illuminated separately using a micro-lens diffuser until a light dose of $20 \mathrm{~J} \cdot \mathrm{cm}^{-2}$ was accomplished. In patient $\mathrm{J}$, three different regions within the target area were illuminated, whereas in de last illumination the isotropic detector probe was removed after $40 \mathrm{~s}$ because it interfered with the exposure of light at the target area.

\subsection{Temporal variation}

All measurements showed major fluence rate fluctuations for a single isotropic detector probe over time. These fluctuations occur despite the surgeons attempt to keep the probe stable within the target area. An example of the fluence and fluence rate over time measured within one isotropic detector probe is shown in Fig. 3. The largest difference between the minimal and maximal measured fluence rate during light exposure was $328 \mathrm{~mW} \cdot \mathrm{cm}^{-2}$. An overview of the measured fluence rate range and mean per isotropic detector probe is shown in Table 2. The aim was to stably illuminate with approximately $80-120 \mathrm{~mW} \cdot \mathrm{cm}^{-2}$ at the target area. In only one probe a mean fluence rate within this range was measured (probe 7 , mean fluence rate $\sim 103 \mathrm{~mW} \cdot \mathrm{cm}^{-2}$ ). However, this fluence rate was reached with an extreme duration of light 
Patient F
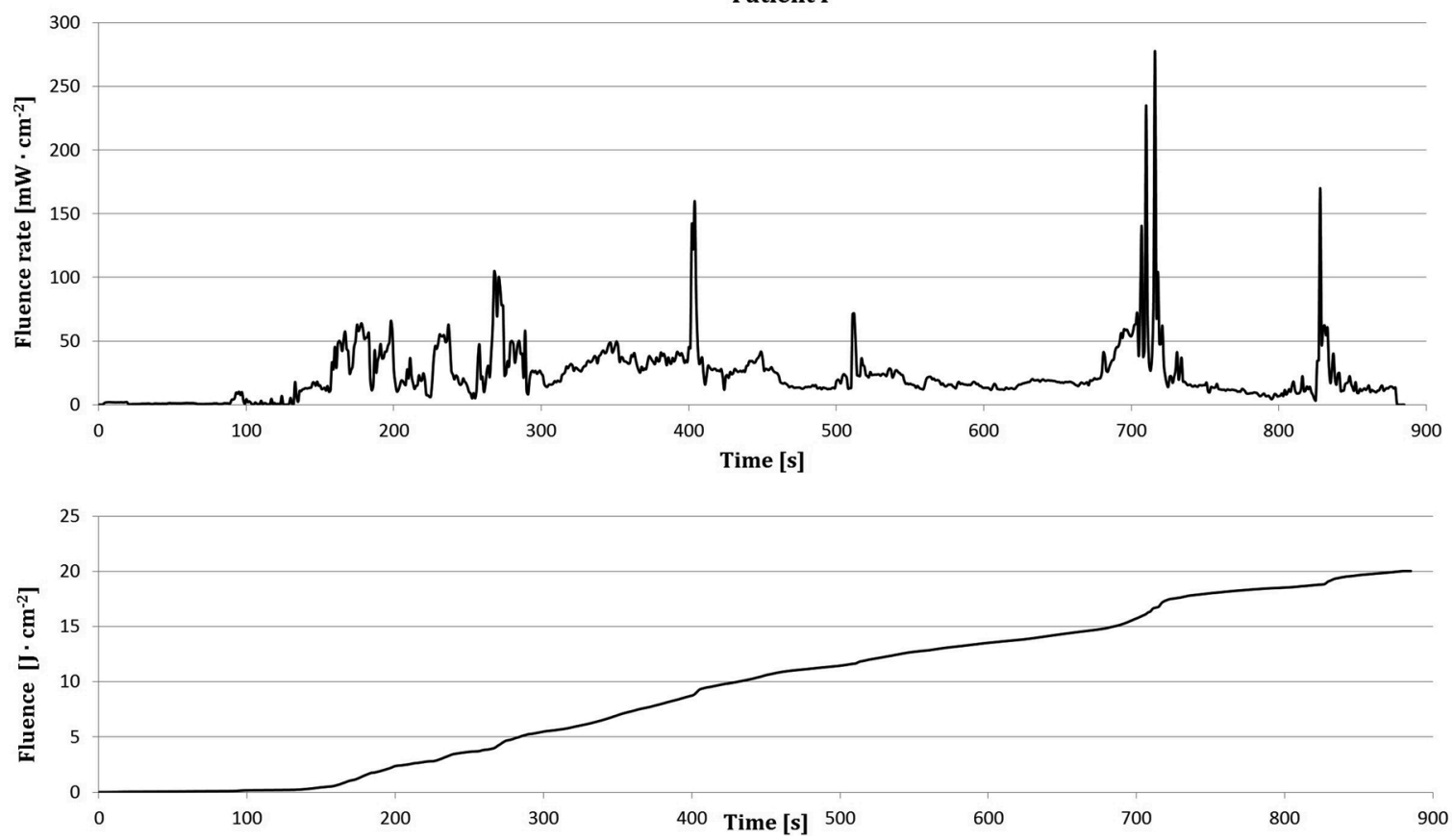

Fig. 3. Graph above: The measured fluence rate (Y-axis) plotted over time (X-axis) within one isotropic detector in patient F. This demonstrates that the fluence rate does not show a stable straight line but many fluctuations over time, so there is a large temporal variability. Graph below: The fluence (i.e. light dose) on the Y-axis, plotted over time (X-axis). The illumination was stopped if the aimed fluence rate of $20 \mathrm{~J} \cdot \mathrm{cm}^{-2}$ was reached.

Table 2

An overview of the measured in vivo light dosimetry characteristics per isotropic detector probe.

\begin{tabular}{|c|c|c|c|c|c|c|c|c|c|}
\hline $\begin{array}{l}\text { Isotropic detector } \\
\text { probe number and } \\
\text { patient letter }\end{array}$ & $\begin{array}{l}\text { Light exposure } \\
\text { duration } \\
\text { seconds }\end{array}$ & $\begin{array}{l}\text { Fluence } \\
\mathrm{J} \cdot \mathrm{cm}^{-2}\end{array}$ & $\begin{array}{l}\text { Minimal } \\
\text { fluence rate } \\
\mathrm{mW} \cdot \mathrm{cm}^{-2}\end{array}$ & $\begin{array}{l}\text { Maximal } \\
\text { fluence rate } \\
\mathrm{mW} \cdot \mathrm{cm}^{-2}\end{array}$ & $\begin{array}{l}\text { Range in } \\
\text { fluence rate } \\
\mathrm{mW} \cdot \mathrm{cm}^{-2}\end{array}$ & $\begin{array}{l}\text { Mean } \\
\text { fluence rate } \\
\mathrm{mW} \cdot \mathrm{cm}^{-2}\end{array}$ & $\begin{array}{l}\text { Standard } \\
\text { deviation } \\
\mathrm{mW} \cdot \mathrm{cm}^{-2}\end{array}$ & $\begin{array}{l}\text { Mean fluence rate } \\
\text { after } 200 \mathrm{~s} \text { light } \\
\text { exposure } \\
\mathrm{mW} \cdot \mathrm{cm}^{-2}\end{array}$ & $\begin{array}{l}\text { Fluence after } \\
200 \mathrm{~s} \text { light } \\
\text { exposure } \\
\mathrm{J} \cdot \mathrm{cm}^{-2}\end{array}$ \\
\hline $1, \mathrm{~A}$ & 301 & 20.3 & 26.7 & 130.1 & 103.4 & 67.4 & 32.8 & 48.1 & 9.6 \\
\hline $2, \mathrm{~A}$ & 301 & 18.9 & 18.4 & 102.5 & 84.1 & 62.7 & 21.9 & 69.7 & 14.0 \\
\hline $3, \mathrm{~B}$ & 760 & 10.8 & 0.1 & 56.5 & 56.4 & 13.5 & 8.9 & 10.2 & 2.1 \\
\hline 4, C & 164 & 10.7 & 53.6 & 123.8 & 70.2 & 65.4 & 14.7 & 65.4 & 10.7 \\
\hline $5, \mathrm{C}$ & 164 & 10.0 & 53.4 & 75.2 & 21.7 & 61.2 & 6.1 & 61.2 & 10.0 \\
\hline $6, \mathrm{D}$ & 200 & 24.6 & 46.1 & 197.6 & 151.5 & 122.9 & 33.0 & 122.9 & 24.6 \\
\hline 7, E & 2283 & 236.2 & 8.3 & 289.7 & 281.4 & 103.3 & 50.9 & 158.6 & 31.7 \\
\hline $8, F$ & 747 & 20.0 & 2.4 & 277.8 & 275.4 & 26.5 & 22.1 & 30.3 & 6.3 \\
\hline $9, \mathrm{G}$ & 565 & 7.4 & 8.2 & 33.5 & 25.3 & 13.1 & 3.7 & 15.9 & 3.2 \\
\hline $10, \mathrm{G}$ & 565 & 20.2 & 18.1 & 59.9 & 41.9 & 35.7 & 6.6 & 30.4 & 6.1 \\
\hline $11, \mathrm{H}$ & 163 & 28.9 & 110.9 & 218.8 & 107.9 & 176.6 & 20.4 & 176.6 & 28.7 \\
\hline $12, \mathrm{H}$ & 163 & 20.1 & 51.3 & 160.9 & 109.7 & 123.6 & 15.7 & 123.6 & 20.1 \\
\hline $13, \mathrm{I}$ & 804 & 23.9 & 0.1 & 154.8 & 154.7 & 29.2 & 22.9 & 22.4 & 4.6 \\
\hline $14, \mathrm{~J}$ & 471 & 35.2 & 17.6 & 345.4 & 327.8 & 74.0 & 44.9 & 105.8 & 21.2 \\
\hline $15, \mathrm{~K}$ & 400 & 6.3 & 4.4 & 21.4 & 17.0 & 15.8 & 4.9 & 12.4 & 2.5 \\
\hline
\end{tabular}

exposition (2283 $\mathrm{s}$ ) and a large range with a minimal measured fluence rate of $\sim 8 \mathrm{~mW} \cdot \mathrm{cm}^{-2}$ and maximal fluence rate of $\sim 289 \mathrm{~mW} \cdot \mathrm{cm}^{-2}$. Probe 10 showed the most stable measured fluence rate, in circa 87 percent of the illumination time the fluence rate was between 20-40 $\mathrm{mW} \cdot \mathrm{cm}^{-2}$. In addition, probe 5 showed fairly stable fluence rates, all within $40-80 \mathrm{~mW} \cdot \mathrm{cm}^{-2}$. See Table 3 for the percentage measured fluence rate per isotropic detector probe over time. In all isotropic detector probes taken together, there was in $22 \%$ of the time a fluence rate measured of less than $20 \mathrm{~mW} \cdot \mathrm{cm}^{-2}$. In $31 \%$ of the illumination time of all probes, a fluence rate above the $80 \mathrm{~mW} \cdot \mathrm{cm}^{-2}$ was measured. An overview of the fluence rates divided into categories of $40 \mathrm{~mW} \cdot \mathrm{cm}^{-2}$ over the light exposure time can be seen in Fig. 4. Usually when treating head and neck malignancies with PDT, the light exposure time is set to $200 \mathrm{~s}$ at $100 \mathrm{~mW} \cdot \mathrm{cm}^{-2}$. In this cohort in $33 \%$ (5 probes) a light dose of at least $20 \mathrm{~J} \cdot \mathrm{cm}^{-2}$ was measured after $200 \mathrm{~s}$ of illumination. Which means that if no dosimetry was used in these patients and the light exposition durance was set to $200 \mathrm{~s}$, the target area in the majority would have been underexposed (less than $20 \mathrm{~J} \cdot \mathrm{cm}^{-2}$ ).

\subsection{Spatial variation}

In four patients two individual probes were used to measure the fluence rate at two different locations. In all patients there was a large spatial variation between the measured fluence rates at the two different positions within the cavity. The fluence rate course of the two probes of patient A, C, G and $\mathrm{H}$ is shown in Fig. 5. A reasonable stable fluence rate was measured for a longer period at the end of the illumination in patient $\mathrm{C}$ and $\mathrm{G}$. The greatest variability in fluence rate in both isotropic detector probes was seen in patient $\mathrm{A}$. In patient $\mathrm{G}$ there may be a slight negative correlation visible, showing that if the fluence rate in probe 9 decreases, the fluence rate in probe 10 increased. 
Table 3

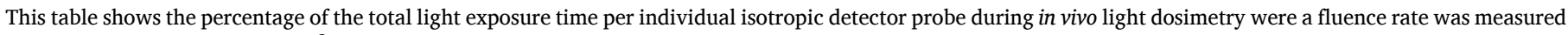
within categories of $40 \mathrm{~mW} \cdot \mathrm{cm}^{-2}$.

\begin{tabular}{|c|c|c|c|c|c|c|}
\hline $\begin{array}{l}\text { Isotropic detector probe } \\
\text { number and patient letter }\end{array}$ & $\begin{array}{l}\text { Fluence rate } 0-40 \\
\mathrm{~mW} \cdot \mathrm{cm}^{-2} \\
\text { (\%time) }\end{array}$ & $\begin{array}{l}\text { Fluence rate } 41-80 \\
\mathrm{~mW} \cdot \mathrm{cm}^{-2} \\
(\% \text { time) }\end{array}$ & $\begin{array}{l}\text { Fluence rate } 81-120 \\
\mathrm{~mW} \cdot \mathrm{cm}^{-2} \\
\text { (\%time) }\end{array}$ & $\begin{array}{l}\text { Fluence rate } 121-160 \\
\mathrm{~mW} \cdot \mathrm{cm}^{-2} \\
(\% \text { time })\end{array}$ & $\begin{array}{l}\text { Fluence rate } 161-200 \\
\mathrm{~mW} \cdot \mathrm{cm}^{-2} \\
\text { (\%time) }\end{array}$ & $\begin{array}{l}\text { Fluence rate }>200 \\
\mathrm{~mW} \cdot \mathrm{cm}^{-2} \\
\text { (\%time) }\end{array}$ \\
\hline $1, \mathrm{~A}$ & 24 & 36 & 39 & 1 & - & - \\
\hline $2, \mathrm{~A}$ & 21 & 54 & 25 & - & - & - \\
\hline $3, \mathrm{~B}$ & 97 & 3 & - & - & - & - \\
\hline $4, C$ & - & 86 & 13 & 1 & - & - \\
\hline $5, \mathrm{C}$ & - & 100 & - & - & - & - \\
\hline $6, \mathrm{D}$ & - & 1 & 63 & 20 & 16 & \\
\hline $7, \mathrm{E}$ & 15 & 25 & 15 & 30 & 14 & 1 \\
\hline $8, F$ & 84 & 14 & 1 & 1 & - & - \\
\hline $9, \mathrm{G}$ & 100 & - & - & - & - & - \\
\hline $10, \mathrm{G}$ & 88 & 12 & - & - & - & - \\
\hline $11, \mathrm{H}$ & - & - & 1 & 16 & 70 & 13 \\
\hline $12, \mathrm{H}$ & - & 1 & 36 & 62 & 1 & - \\
\hline $13, \mathrm{I}$ & 77 & 19 & 3 & 1 & - & - \\
\hline $14, \mathrm{~J}$ & 16 & 47 & 31 & 4 & - & 2 \\
\hline $15, \mathrm{~K}$ & 100 & - & - & - & - & - \\
\hline
\end{tabular}

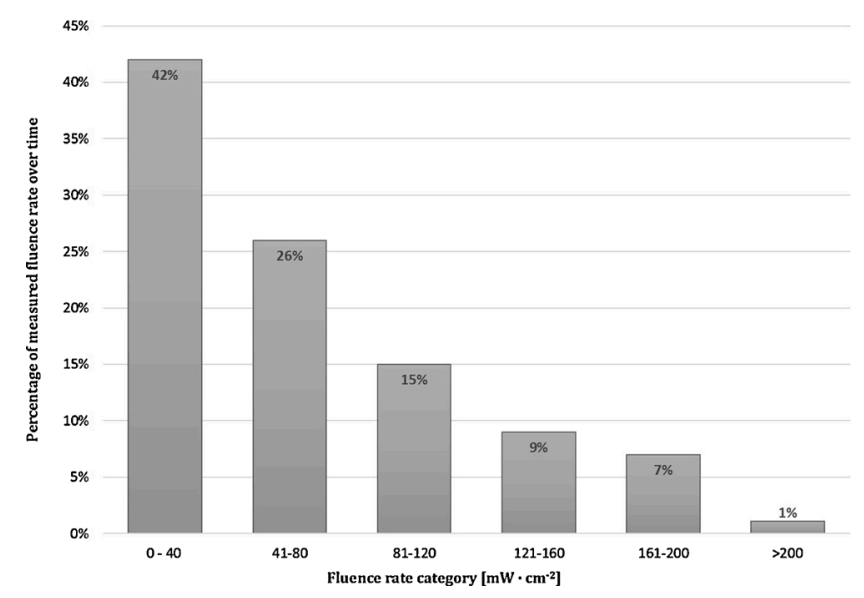

Fig. 4. An overview of the percentage of measured fluence rate of the total illumination time in all isotropic detector probes taken together. The fluence rate is divided in categories of $40 \mathrm{~mW} \cdot \mathrm{cm}^{-2}$ size.

\section{Discussion}

In this clinical dosimetric study during sinonasal mTHPC-mediated PDT, large temporal and spatial variations in fluence rate were observed. In which it proved to be very challenging to deliver a stable, homogeneous and desired fluence rate and fluence to the target area as expected according to previous research [10,12-15,19,24]. According to the European Medicine Agency patients were treated with a mTHPC dose of $0.15 \mathrm{~kg} / \mathrm{mg}$ and a drug-time interval of 4 days. Previous studies have shown that the time-interval as well as different drug dose may improve the PDT treatment effect and show less toxicity to the patient [25-28]. However, drug dose as well as drug-light interval were outside the scope of this study. There are a number of limitations, when using the current dosimetry and PDT approach, which affect these variations.

First, the dosimetric approach used merely utilizes a simple, first order calculation of the expected direct incident fluence rate for an estimated 'optimal' source location in relation to the target area. This method was in particular based on the source output and the distance between the light source and the target area, determined using CT or MR imaging. A number of essential influential aspects were not taken into account within this approach including: the optical properties with scattering and absorption values, the complex geometry of the paranasal cavity with grooves, niches and corners, the influence of back-scattering with the associated fluence rate build-up and 3D light distribution

\section{[12-14,29].}

Additionally, an attempt was made to reach the pre-determined preferred light source location during the PDT manually by the surgeon. However, the only feedback in reaching this optimal location is the inaccurate use of the two dimensional endoscopic view. Furthermore, the manual positioning causes, despite the surgeon's steady hand, small movements resulting in variation in distance between the source and probes and therefore fluence rate variations. The use of reflecting elastic balloons with an incorporated light source are found to be of added value in PDT $[17,30,31]$. The possibility for use within the paranasal sinuses has yet to be explored, but might be challenging due to the wide variation of the sinus anatomy, especially after salvage surgery. In case of an elongated smooth cylindrical cavity, a linear diffuser with balloon applicator could be considered. However, the balloon might put pressure on the mucosa, thereby compressing the capillaries, hindering the oxygen supply necessary for PDT.

In addition, an attempt was made to place the isotropic detectors in the target area, ideally in direct view of the light source with minimal interference with the light exposure in the target area. Due to the anatomy and the passage of the isotropic detector probes via the nasal cavity, parallel to the light source and endoscope, this results in a sideway position of the probe related to the light source, which might lead to an under estimation of the actual fluence rate. Fig. 4 shows that in this study the majority of measured fluence rate was below the 80 $\mathrm{mW} \cdot \mathrm{cm}^{-2}$. Overall, the current approach lacks in reliable quantitative dosimetric feedback and verification of the actual position of, and distances between the light source, isotropic detector probe(s) and target area.

Moreover, there are intra- and interpatient variations in optical properties whereas the paranasal cavity surface is irregular and consisting of different coverings such as intact mucosa, affected mucosa, scar tissue, crusts and exposed bone [5,32-34]. In addition, during the light exposure, the optical properties can change due to instant vascular effects of the PDT $[3,19,35]$ and thus results in an increase or decrease in measured fluence rate.

The spatial variations are difficult to quantify due to the complexity of the sinonasal geometry and the aforementioned heterogeneous distribution of the optical properties. Besides, conclusions in spatial variation could not be drawn because the exact distances between the light source, the isotropic detector probes and target area were unknown. In addition, a maximum of two measuring isotropic probes within the cavity is not sufficient to get a reliable impression of the global light distribution in the 3D space of the sinonasal cavity, as the probes were mainly positioned in, or in the vicinity of the target area.

Finally, due to the limited patient numbers, variability of geometry and optical properties within the target location, light diffuser type 

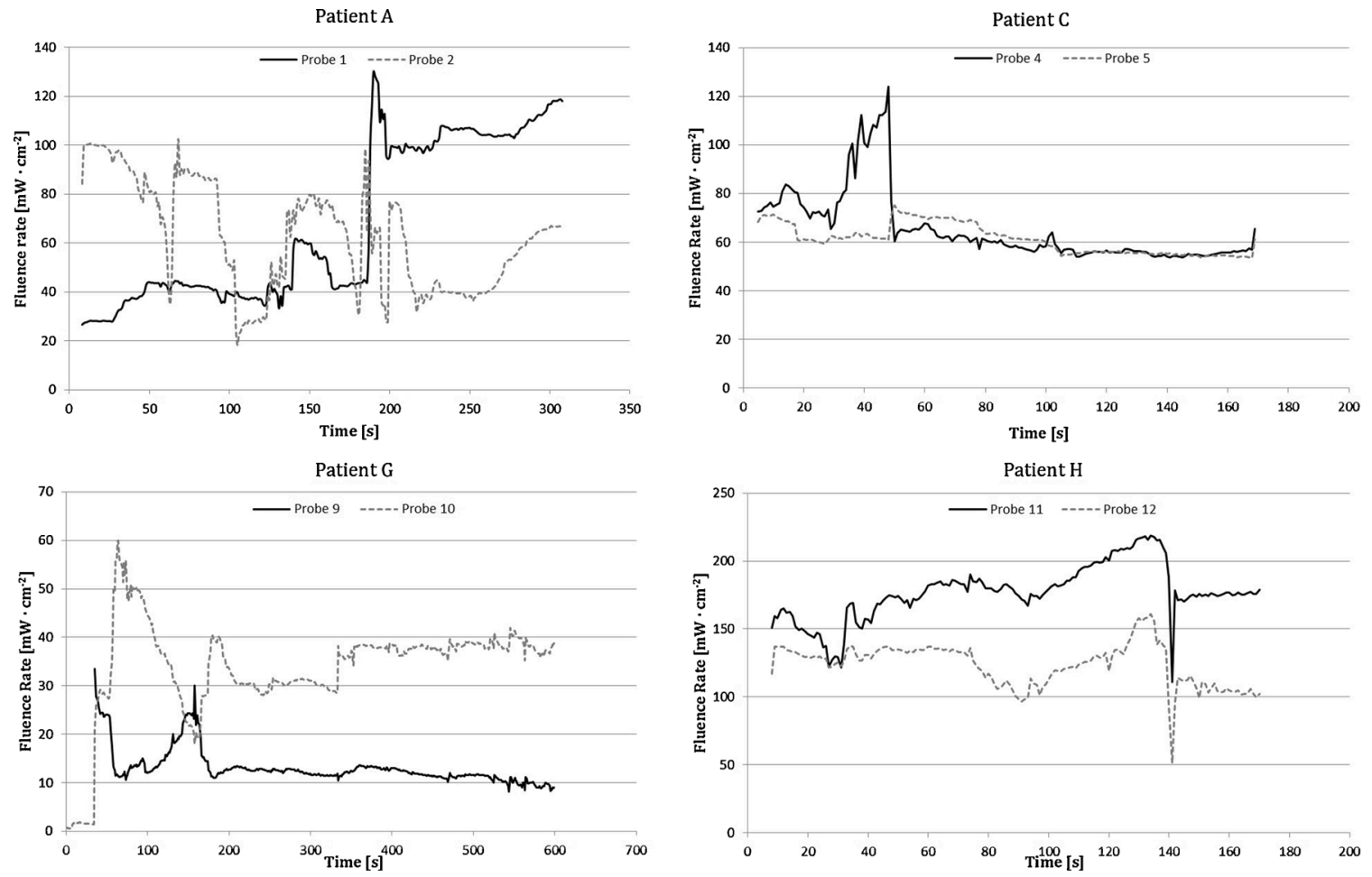

Fig. 5. The fluence rate (Y-axis) over time (X-axis) measured by two individual isotropic detector probes positioned at two different locations within the same target area, in four different patients (Patient A, C, G and H).

employed and source output used, no definitive conclusions regarding the exact origin of the observed variation in fluence rate can be drawn.

Also in view of the limited number of patients and aforementioned variations, no statement could be made in this cohort about the clinical response to the PDT in relation to the fluence rate.

\section{Conclusion \& future perspectives}

In conclusion, the current paranasal sinus PDT dosimetry approach demonstrated major temporal and spatial variations in fluence rate and fluence. Despite the promising clinical results [20,22], the current approach is not reproducible, neither standardized and inaccurate. On account of manually positioning of the light source and isotropic detector probes, and an inaccurate pre-treatment planning of the desired source location.

In light of these limitations, our group is directing future research to develop a reliable dosimetric approach that allows for sinonasal dosimetry pre-planning in 3D. This model will take the tissue back scattering i.e. tissue optical properties into account to determine the most optimal source location(s), output power and light diffuser type [36]. Previous research demonstrated that the fluence rate distribution can be determined in tissue optical phantoms that closely match the target area geometry and optical properties [6,19]. Our developed 3D models are therefore tested and based on 3D phantoms with a patient-specific paranasal sinus geometry and known optical properties. Employing electromagnetic navigation can assist in positioning the isotropic detector probes and light source to the pre-determined optimal locations and allows direct visual feedback of the actual positions during the light exposure [37]. Besides, a fixation tool will be developed to enable a stable fluence rate delivery, whereas several studies showed a reduction of instrument movements by using a surgical robot device compared to manual surgery [38,39]. In addition, the in vivo optical properties of the paranasal sinus will be determined by means of multidiameter single-fiber reflectance (MDSFR) spectroscopy; these properties will be incorporated in this developed 3D light distribution model.

\section{Source of financial support or funding}

This study is partially funded by Biolitec ${ }^{\circledR}$ (Jena, Germany).

\section{Declaration of Competing Interest}

The authors report no declarations of interest.

\section{References}

[1] B.W. Henderson, T.M. Busch, J.W. Snyder, Fluence rate as a modulator of PDT mechanisms, Lasers Surg. Med. 38 (5) (2006) 489-493.

[2] L.H. Murrer, et al., Short- and long-term normal tissue damage with photodynamic therapy in pig trachea: a fluence-response pilot study comparing Photofrin and mTHPC, Br. J. Cancer 80 (5-6) (1999) 744-755.

[3] B. Kruijt, et al., Monitoring interstitial m-THPC-PDT in vivo using fluorescence and reflectance spectroscopy, Lasers Surg. Med. 41 (9) (2009) 653-664.

[4] T.M. Busch, et al., Photodynamic therapy creates fluence rate-dependent gradients in the intratumoral spatial distribution of oxygen, Cancer Res. 62 (24) (2002) $7273-7279$.

[5] M.M. Kim, A. Darafsheh, Light sources and dosimetry techniques for photodynamic therapy, Photochem. Photobiol. (2020).

[6] R. Bays, et al., Light dosimetry for photodynamic therapy in the esophagus, Lasers Surg. Med. 20 (3) (1997) 290-303.

[7] S.A. Blant, et al., In vivo fluence rate effect in photodynamic therapy of early cancers with tetra(m-hydroxyphenyl)chlorin, Photochem. Photobiol. 64 (6) (1996) 963-968.

[8] A.K. D'Cruz, M.H. Robinson, M.A. Biel, mTHPC-mediated photodynamic therapy in patients with advanced, incurable head and neck cancer: a multicenter study of 128 patients, Head Neck 26 (3) (2004) 232-240.

[9] M.A. Biel, Photodynamic therapy of head and neck cancers, Methods Mol. Biol. 635 (2010) 281-293.

[10] T.C. Zhu, J.C. Finlay, The role of photodynamic therapy (PDT) physics, Med. Phys. 35 (7) (2008) 3127-3136.

[11] I.B. Tan, et al., Temoporfin-mediated photodynamic therapy in patients with advanced, incurable head and neck cancer: a multicenter study, Head Neck 32 (12) (2010) 1597-1604.

[12] R.L. van Veen, et al., In vivo fluence rate measurements during Foscan-mediated photodynamic therapy of persistent and recurrent nasopharyngeal carcinomas using a dedicated light applicator, J. Biomed. Opt. 11 (4) (2006) 041107.

[13] J.P. Marijnissen, et al., In situ light dosimetry during whole bladder wall photodynamic therapy: clinical results and experimental verification, Phys. Med. Biol. 38 (5) (1993) 567-582. 
[14] R.L. van Veen, et al., In situ light dosimetry during photodynamic therapy of Barrett's esophagus with 5-aminolevulinic acid, Lasers Surg. Med. 31 (5) (2002) 299-304.

[15] I.B. Tan, et al., The importance of in situ light dosimetry for photodynamic therapy of oral cavity tumors, Head Neck 21 (5) (1999) 434-441.

[16] H.J. van Staveren, et al., Integrating sphere effect in whole-bladder wall photodynamic therapy: III. Fluence multiplication, optical penetration and light distribution with an eccentric source for human bladder optical properties, Phys. Med. Biol. 41 (4) (1996) 579-590.

[17] W. Beyer, Systems for light application and dosimetry in photodynamic therapy, J. Photochem. Photobiol. B 36 (2) (1996) 153-156.

[18] N. Betrouni, et al., Real-time light dosimetry for intra-cavity photodynamic therapy: application for pleural mesothelioma treatment, Photodiagnosis Photodyn. Ther. 18 (2017) 155-161.

[19] H.J. Nyst, et al., Performance of a dedicated light delivery and dosimetry device for photodynamic therapy of nasopharyngeal carcinoma: phantom and volunteer experiments, Lasers Surg. Med. 39 (8) (2007) 647-653.

[20] L. Caesar, et al., The use of photodynamic therapy as adjuvant therapy to surgery in recurrent malignant tumors of the paranasal sinuses, Photodiagnosis Photodyn. Ther. 12 (3) (2015) 414-421.

[21] B. Karakullukcu, et al., A matched cohort comparison of mTHPC-mediated photodynamic therapy and trans-oral surgery of early stage oral cavity squamous cell cancer, Eur. Arch. Otorhinolaryngol. 270 (3) (2013) 1093-1097.

[22] T.E.M. van Doeveren, et al., Adjuvant photodynamic therapy in head and neck cancer after tumor-positive resection margins, Laryngoscope 128 (3) (2018) 657-663.

[23] www.ema.europa.eu/foscan.

[24] T.C. Zhu, A. Lu, Y.H. Ong, An improved analytic function for predicting light fluence rate in circular fields on a semi-infinite geometry, Proc. SPIE. Int. Soc. Opt. Eng. 9706 (2016) 97061D.

[25] D.R. Braichotte, et al., Clinical pharmacokinetic studies of photofrin by fluorescence spectroscopy in the oral cavity, the esophagus, and the bronchi, Cancer 75 (11) (1995) 2768-2778.

[26] G. Wagnieres, et al., Clinical evaluation of the cutaneous phototoxicity of 5,10,15,20-tetra(m-hydroxyphenyl)chlorin, Photochem. Photobiol. 68 (3) (1998) $382-387$.
[27] M. Zellweger, et al., Stability of the fluorescence measurement of Foscan in the normal human oral cavity as an indicator of its content in early cancers of the esophagus and the bronchi, Photochem. Photobiol. 69 (5) (1999) 605-610.

[28] T. Glanzmann, et al., Pharmacokinetics and pharmacodynamics of tetra(mhydroxyphenyl)chlorin in the hamster cheek pouch tumor model: comparison with clinical measurements, J. Photochem. Photobiol. B 57 (1) (2000) 22-32.

[29] L.H. Murrer, H.P. Marijnissen, W.M. Star, Monte Carlo simulations for EndoBronchial Photodynamic Therapy: the influence of variations in optical and geometrical properties and of realistic and eccentric light sources, Lasers Surg. Med. 22 (4) (1998) 193-206.

[30] H. van den Bergh, On the evolution of some endoscopic light delivery systems for photodynamic therapy, Endoscopy 30 (4) (1998) 392-407.

[31] L. Gossner, et al., A new long-range through-the-scope balloon applicator for photodynamic therapy in the esophagus and cardia, Endoscopy 31 (5) (1999) 370-376.

[32] J.L. Sandell, T.C. Zhu, A review of in-vivo optical properties of human tissues and its impact on PDT, J. Biophotonics 4 (11-12) (2011) 773-787.

[33] Y.H. Ong, et al., Determination of optical properties, drug concentration, and tissue oxygenation in human pleural tissue before and after Photofrin-mediated photodynamic therapy, Proc. SPIE. Int. Soc. Opt. Eng. 10476 (2018).

[34] B. Karakullukcu, et al., Clinical feasibility of monitoring m-THPC mediated photodynamic therapy by means of fluorescence differential path-length spectroscopy, J. Biophotonics 4 (10) (2011) 740-751.

[35] P. Cramers, et al., Foscan uptake and tissue distribution in relation to photodynamic efficacy, Br. J. Cancer 88 (2) (2003) 283-290.

[36] T.E.M. van Doeveren, et al., On the Development of a Light Dosimetry Planning Tool for Photodynamic Therapy in Arbitrary Shaped Cavities: Initial Results, Photochem. Photobiol. (2020).

[37] M.M. Kim, et al., Infrared navigation system for light dosimetry during pleural photodynamic therapy, Phys. Med. Biol. (2020).

[38] D.A.L. Maberley, et al., A comparison of robotic and manual surgery for internal limiting membrane peeling, Graefes Arch. Clin. Exp. Ophthalmol. (2020).

[39] T.J.M. van Mulken, et al., First-in-human robotic supermicrosurgery using a dedicated microsurgical robot for treating breast cancer-related lymphedema: a randomized pilot trial, Nat. Commun. 11 (1) (2020) 757. 